\title{
AN IMPROVED EYE DETECTION METHOD BASED ON STATISTICAL MOMENTS
}

\author{
${ }^{1}$ Saideh Ferdowsi, ${ }^{2}$ Vahid Abolghasemi, ${ }^{1}$ Alireza Ahmadyfard, and ${ }^{2}$ Saeid Sanei \\ ${ }^{1}$ Faculty of Electrical \& Robotic Engineering, Shahrood University, Shahrood, IRAN \\ ${ }^{2}$ Center of Digital Signal Processing, School of Engineering, Cardiff University, Cardiff, UK \\ ${ }^{1}$ saideh_ferdosi@yahoo.com, ${ }^{1}$ ahmadyfard@ shahroodut.ac.ir, ${ }^{2}$ \{abolghasemiv,saneis\}@cf.ac.uk
}

\begin{abstract}
In this paper the problem of eye detection in $2 \mathrm{D}$ grayscale images is addressed. The proposed method analyses the input face images in topographic format. The reason is to alleviate sensitivity of the algorithm to illumination and contrast changes. Invariant moments are used as robust features describing eye shape. A new strategy to select robust features based on their variance among training images is proposed. Using several complementary features such as existing of nose between eyes, some non-eye candidates are removed. Finally, a Bayesian classifier is used to select the most probable locations of eyes. The eye detection results show a higher detection rate and robustness compared to the existing methods. The performance rate has increased comparing to our previous algorithm presented in [4].
\end{abstract}

Index Terms - Eye detection, Hessian matrix, Eigenvalue decomposition, Moment invariants.

\section{INTRODUCTION}

In many face recognition problems detecting eye locations has been investigated prior to other processes because of relative stability and strength of this feature. Beside the application of eye detection in recognition tasks, there are a wide range of applications such as driver fatigue detection [1], Human Computer Interface (HCI) [2], video conferencing, and disabled people aiding systems. However, there are some problems in finding the exact location of eyes which cause this task to be difficult. Dealing with different eye sizes, positions of head, eye closing, eye blinks, lighting conditions, and occlusion by hair and frame of glasses are some of the most important issues that should be considered when designing a robust eye detection algorithm. What follows is a description of previous works regarding their strengths and weaknesses in eye detection.

A general eye model is required as the first step in template based methods. Then, a template matching technique is used to search for the eye locations. Template based methods are highly sensitive to the model parameters and the initialization. In feature based methods one attempts to extract some distinctive features around eyes using the observations such as edge and intensity of iris, eye corners, the color distributions of the sclera and the flesh in the image [3]. In these methods problem arises when dealing with closed eyes or partially occluded by hair or glasses or due to face orientation [4]. The last class of eye detection methods are appearance based methods. In this approach the aim is to learn the eye photometric appearance using a learning procedure of eye images [4]. Then, the learnt system is used to search for presence of eyes in a test image. A large amount of training data under different face orientations and different illumination conditions are required in order to well represent the eyes in different images.

Recently, there have been some efforts to use topographic 3D surface of eye as a robust feature to illumination change for detection [4] [5]. In [5] Wang et al. attempt to derive terrain map from a gray level image based on the topographic primal sketch theory and then use a terrain feature matching algorithm for eye detection and tracking. Then, Ferdowsi et al. [4] used invariant moments to describe eye shapes and increasing the detection rate.

In this paper a modified version of the method presented in [4] is proposed. The contribution is in improving the feature vector extracted from eye patches. Here, instead of using all features obtained from topographic labels we use only features of those labels which have smaller variances. Moreover, based on some geometrical characteristics such as distance between eyes and the orientation of the eye-pair against the horizontal axis which need to be in a certain range, some candidates are rejected. In addition, existence of nose as a new complementary characteristic is employed. Finally, in the proposed method the Bayesian classifier considers left and right classes only, and ignores the noneye class. The reason is that high variability of non-eye features avoids a clear description of a non-eye class. The results show the strength of the proposed method in detecting the eye locations.

The rest of the paper is as follows. Section 2 describes different steps to find topographic labels. In section 3 invariant moments for making feature vectors are computed. Section 4 proposes a strategy to select effective features. Eye detection is finalized using a Bayesian classifier in section 5. Experimental results are given in section 6 . Finally, the paper is concluded in section 7. 


\section{TOPOGRAPHIC LABELS}

If we sketch a $3 D$ representation of eye surface using a gray scale image in which $X$ and $Y$ are pixel locations and $Z$ is the intensity of each pixel, we observe that the pupil of human eye is located in a local minimum (Fig. 1). Since a local minimum in pupil is a very robust feature for which we need to employ an appropriate map independent of intensity with small number of descriptors [5]. For this purpose a topographic terrain map is employed in which each pixel in the grayscale image with 256 possible values is mapped to one of the twelve existing labels which have been shown in Fig. 2. This can help us to detect eye locations by exploring their terrain features.

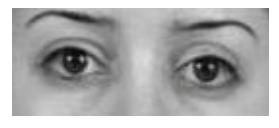

(a)

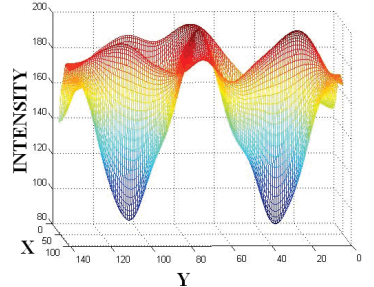

(b)
Fig. 1 (a) Gray-scale image and (b) its 3D representation

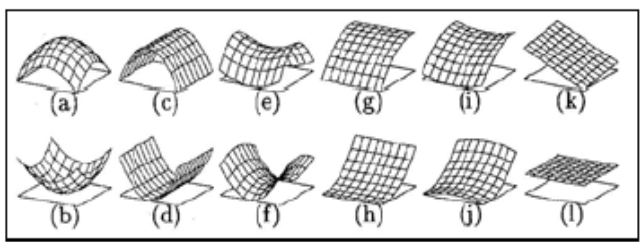

Fig. 2 Topographic labels (a) peak (b) pit (c) ridge (d) ravine (e) ridge saddle (f) ravine saddle $(\mathrm{g})$ convex hill (h) concave hill (i) convex saddle hill (j) concave saddle hill (k) slop hill and (l) flat

In order to obtain the values of topographic labels we need to calculate first and second order derivatives of the input image. The Hessian matrix of the input intensity image is denoted by $f(x, y)$ is defined as:

$$
\boldsymbol{H}=\left[\begin{array}{ll}
\frac{\partial^{2} f(x, y)}{\partial x^{2}} & \frac{\partial^{2} f(x, y)}{\partial x \partial y} \\
\frac{\partial^{2} f(x, y)}{\partial x \partial y} & \frac{\partial^{2} f(x, y)}{\partial y^{2}}
\end{array}\right]
$$

Since $f(x, y)$ is a digital image and not a continuous function one cannot calculate the derivatives, rather needs to estimate them. In order to do this, we employ a smoothed differentiation filter based on the Chebyshev polynomial [6]. The $p$ th and $q$ th order derivatives of $f(x, y)$ with respect to variables $x$ and $y$ respectively is estimated as:

$f^{(p, q)}(x, y)=\sum_{i=-N}^{N} \sum_{j=-N}^{N} f(x-i, y-j) h(i, p) h(j, q)$

Where $f(x, y)$ is the digital input image. Filters $h(i, p)$ and $h(j, q)$ are kernels for estimation of function derivatives along $x$ and $y$ directions using Chebyshev polynomials [6].
In order to determine the topographic labels we need to calculate the eigenvalues, eigenvectors of the Hessian matrix, and the vector of surface gradient at each pixel. This can be done using eigenvalue decomposition method described by:

$$
H=U D U^{T}=\left[\begin{array}{ll}
u_{1} & u_{2}
\end{array}\right] \operatorname{diag}\left(\begin{array}{ll}
\lambda_{1} & \lambda_{2}
\end{array}\right)\left[\begin{array}{ll}
u_{1} & u_{2}
\end{array}\right]^{T}
$$

Where $\lambda_{1}$ and $\lambda_{2}$ are the eigenvalues and $u_{1}$ and $u_{2}$ are the corresponding eigenvectors, and (.) ${ }^{T}$ denotes transpose operation. Further details on determining the topographic labels can be obtained by referring to [4] and [5].

Fig. 3 shows the topographic map in eye region. In Fig. 4 the extracted topographic labels have been given. It can be seen that the topographic map in the eye area is composed of five considerable regions: (d), (g), (h), (i) and (j) which correspond to ravine, convex hill, concave hill, convex saddle hill and concave saddle hill, respectively (Fig. 2). The aim is then to design an appropriate model which truly describes the eye shape using these labels [4]. In the next section the solution to this problem is investigated.

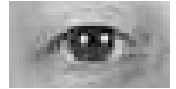

(a)

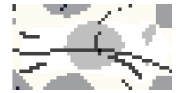

(b)
Fig. 3 (a) A sample eye region and (b) The equivalent topographic map

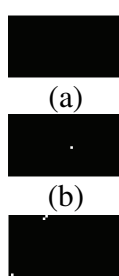

(c)

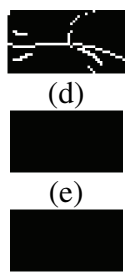

(f)

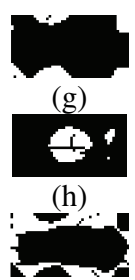

(i)

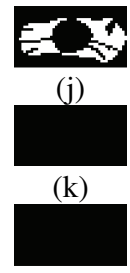

(1)
Fig. 4 Corresponding topographic labels of an eye image extracted from Fig. 3 (b)

\section{MOMENT INVARIANTS}

Moments which are one of the best tools for describing the shape of an object and are invariant to scale, rotation and translation were first introduced by $\mathrm{Hu}$ [7]. In this paper we use seven invariant moments to describe each labeled region in the terrain map. If the corresponding region to $l$ th topographic label is denoted by $R_{l}$, the $(p+q)$ th geometric moment of this region can be defined by:

$$
\begin{array}{lcl}
m_{p q}=\sum_{x=0}^{M-1} \sum_{y=0}^{N-1} x^{p} y^{q} R_{l}(x, y) & p, q=0,1,2, \ldots \\
\bar{x}=\frac{m_{10}}{m_{00}} & \text { and } & \bar{y}=\frac{m_{01}}{m_{00}}
\end{array}
$$


Changing Eq. (4) to:

$$
\mu_{p q}=\sum_{x=0}^{M-1} \sum_{y=0}^{M-1}(x-\bar{x})^{p}(y-\bar{y})^{q} R_{l}(x, y)
$$

gives us the central moment which is invariant to translation [7]. $\mathrm{Hu}$ [7] states if these invariants are combined with the similitude invariants of central moments, then, pattern identification can be made of position, size, and orientation. In other words, the descriptive strength of individual moments are not high enough to uniquely represent arbitrary shapes, while sets of functions based on these moments are highly powerful. Based on these observations, combining results of Eq. (5) with assigning different values of $p$ and $q$, Hu defined seven moments denoted by $\phi_{i} \quad(i=1$, $2 \ldots, 7)$ which can be found in [7].

Since we have already obtained five important topographic labels in the eye region (Fig. 4) and each of which has seven moments (which can be called features), the whole number of features would be $5 \times 7=35$. These moments can be easily put into a feature vector indexed for simplicity by $\vec{f}=\left[\phi_{1}, \phi_{2}, \ldots, \phi_{35}\right]^{T}$.

\section{FEATURE SELECTION}

Studying the values of these features for a large number of eye patches from different face images showed that the values of some features do not change significantly in the overall data set, while there exist some features that have very high variances. This tendency can be expressed regarding the robustness of some features in the eye region. In other words, it is estimated that there are some features which are robust in the eye region and they have almost the same value in all patches taken from a variety of faces. In spite of this, there are also some features that are not robust enough and so their values change severely from one eye patch to another. Based on this idea, in the proposed method some features (instead of all) are selected for later processing. The decision criterion for selecting features is based on their variances among all eye patches. Suppose the number of all eye images is $m$, using the obtained feature vector from the previous section we have $m$ such a feature vectors denoted by $\vec{f}_{1}, \vec{f}_{2}, \ldots, \vec{f}_{m}$. The variance of these features can be obtained as follows:

$$
\sigma^{2}=\frac{1}{m} \sum_{i=1}^{m}\left(\overrightarrow{f_{i}}-\bar{f}\right)^{2}
$$

where $\bar{f}$ is the mean of the $m$ vectors.

Each element in vector $\sigma^{2}$ states the robustness of the related feature. The next step is to search for small elements in $\sigma^{2}$ which correspond to more robust features and make a new feature vector. Obviously, the number of elements of this new feature vector is less than 35 . We select twenty-two features corresponding to twenty-two smallest values in $\sigma^{2}$. However, the decision to select "twenty-two" features is made empirically.

\section{EYE DETECTION}

Suppose we have a test face grayscale image as the input, one needs to do some processing to find the most probable eye location. In this regard, first the topographic map of the input image is extracted. Then, all the pixels with topographic labels of type "pit" as pupil candidates are selected. Each possible pair of pit pixels is considered as eye candidate. Obviously, not all these pairs are proper candidates for eye locations and most of them have to be rejected.

\subsection{Non-eye candidate rejection}

Beside the selected features in previous section, there are also other characteristics which can be used for removing many of the non-eye pixel pits. First, each pair composed of pixels being very apart/close is removed. Second, pixel pairs with a high orientation (against the horizontal axis) are removed. It means that, if we connect two elements of a pair by a straight line, the angle of this line with reference to horizontal axis must be in a limited range. Moreover, observing topographic maps of many face images with different appearances showed that the label "slop hill" (Fig. $2(\mathrm{k})$ ) does not appear in the eye region, while there are many label of this type in hairs. Thus, this strong characteristic is also used to remove many non-eye pairs. Another exclusive feature is the existence of nose between two candidate pairs. Since nose is determined with label of type "ridge" (Fig. 2(c)), a procedure has been developed to filter out the pairs having this property. In order not to lose the eye pair by mistake, a narrow bar is selected between each candidate pair and then the existence of this label is being checked among all remained pixels pairs.

After applying the above constraints, a few candidates with a higher probability of being correct eye pairs are still remained. In order to select one pair with the highest probability of being eye-pair, a simple classification using the features obtained in section 3 is applied.

\subsection{Mahalanobis distance measure}

We apply the same procedure for extracting the preferred features discussed in section 4 for the remained candidates. Assuming there are $n$ remained candidate pairs, the result will be a pair of feature vectors, one for left-eye candidate and one for right-eye $\left(\vec{f}_{L, i}, \vec{f}_{R, i}\right.$ and $\left.i=1,2, \ldots, n\right)$. The Mahalanobis distance of these features from the obtained feature pairs in section 4 (eye class) with the mean of $\left(\vec{m}_{L}, \vec{m}_{R}\right)$ and the covariance matrix of $\left(\sum_{L}, \sum_{R}\right)$ is computed as follows: 


$$
D_{i, j}^{2}=\left(f_{i}-m_{j}\right)^{T} \sum_{j}^{-1}\left(f_{i}-m_{j}\right)
$$

(Where $j=L, R$ and $i=1,2 \ldots, n$ )

It is worth noting that the above mean vector and covariance matrix have been obtained from a training experiment on manually selected eye regions. As we had $n$ pairs of candidates, the result will be $n$ distance pairs $\left(D_{L}^{2}, D_{R}^{2}\right)$. In order to select one pair as the detected eye location, only the ones with minimum distance to the centre of both right-eye and left-eye class is selected. The distance of a pair of patches to the eyes model is defined as:

$$
D_{p a i r, k}^{2}=\sqrt{D_{L, k}^{2}+D_{R, k}^{2}}
$$

\section{EXPERIMENTAL RESULTS}

In order to evaluate the performance of the proposed method an experiment using XM2VTS database [8] involving 1300 face images has been carried out. 400 images of right and left eyes are used for training of the classifier which is used in section 5.2.

In addition, some non-restricted images with different backgrounds and illumination changes have been applied to measure the robustness and strength of the method. The obtained results are promising and confirm the high performance of the proposed method. Fig. 5 shows eye detection results for sample images taken from XM2VTS database [8]. It can be seen that the proposed method can successfully detect the eye locations, while the method presented in [4] failed to detect the eye location in the same images.
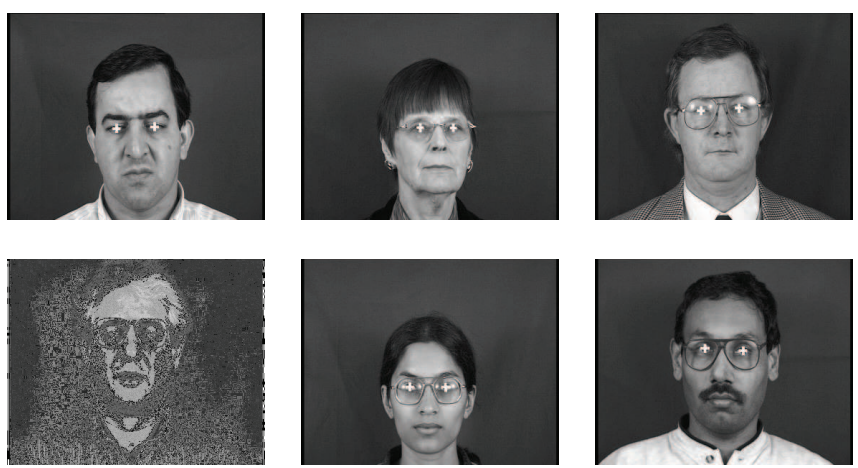

Fig. 5 Eye detection result for some sample face images

In order to evaluate the detection rate and compare the result of our method with those of some recent approaches Table 1 has been formed. As seen from the table, detection rate has been improved comparing to the method presented in [4]. Here, we observe that selecting prominent and robust features in the eye region increases the performance comparing to these when use all extracted features without considering their strengths are used. In addition, searching for existence of nose between the extracted pairs helps to decrease the computation time and also increasing the overall performance.

Table 1. The performance of two eye detection methods

\begin{tabular}{|c|c|c|c|}
\hline Method & Detection rate & $\begin{array}{c}\text { Fail to detect } \\
\text { one eye }\end{array}$ & $\begin{array}{c}\text { Fail to detect } \\
\text { both eyes }\end{array}$ \\
\hline Ref. [4] & $90 \%$ & $7 \%$ & $3 \%$ \\
\hline The proposed & $93.2 \%$ & $4.8 \%$ & $2 \%$ \\
\hline
\end{tabular}

In addition, to faces with plain backgrounds, we also applied the proposed method on some images taken from real scenes with complex backgrounds (Fig. 6). The results are encouraging but still not acceptable and so the method needs more investigation to make it applicable. The method still has difficulty in finding the eye locations in images where the person wears glasses.
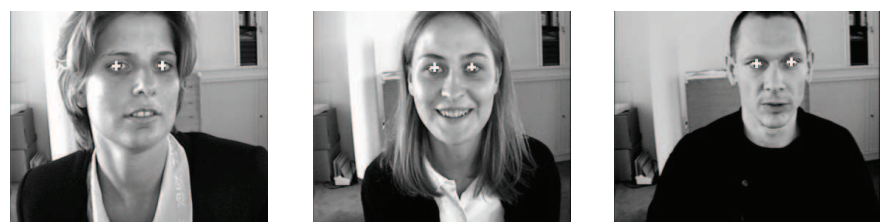

Fig. 6 Eye detection results for face images with complex background

\section{CONCLUSION}

In this paper a modification of the method in [4] has been proposed. The advantage is that here, a feature selection based on variance of feature vectors taken from many training images is proposed. Moreover, some new characteristics such as existence of nose and rejecting labels related to hairs are used for removing the non-eye candidates. The results show a better performance comparing to the previous method.

\section{REFERENCES}

[1] L. Gan, B. Cui, W. Wang, "Driver Fatigue Detection Based on Eye Tracking," in Proc. 6th World Congress on Intelligent Control and Automation, June, 2006.

[2] B. Fasel, J. Luettin, "Automatic facial expression analysis: a survey," Pattern Recognition, vol. 36 pp. 259-275, 2003.

[3] H. Gu, G. Su, and C. Du. "Feature Points Extraction from Faces". Image and Vision Computing Journal. November, 2003.

[4] S. Ferdowsi and A. Ahmadyfard "Using Statistical Moments as Invariants for Eye Detection", in Proc. $16^{\text {th }}$ European Signal Processing Conference EUSIPCO08, Switzerland, August 2008.

[5] J. Wang and L. Yin." Detecting and Tracking Eyes through Dynamic Terrain Feature Matching," in Proc. Computer Vision and Pattern Recognition Conf., CVPR'05, vol. 3, pp. 78 - 78, June, 2005.

[6] P. Meer and I. Weiss, "Smoothed differentiation filters for images," in Proc., 10th International Conference on Pattern Recognition, Maryland, USA, vol. 2, pp. 121-126., Jun, 1990.

[7] M. Hu. "Visual Pattern Recognition by Moment Invariants". IRE Transactions on Information Theory. pp.79-187, 1962.

[8] Provided by "Centre for Vision, Speech and Signal Processing, http://www.ee.surrey.ac.uk/CVSSP/xm2vtsdb/ 